\title{
The dark halo in the spiral galaxy NGC 755
}

\author{
Franz Kenn* \\ RAIUB, Bonn, Germany \\ E-mail: fkenneastro.uni-bonn.de
}

\section{Gyula Józsa}

RAIUB, Bonn, Germany

\section{Gianfranco Gentile}

SISSA, Trieste, Italy

\section{Uli Klein}

RAIUB, Bonn, Germany

\begin{abstract}
The radial density distribution in dark matter halos of galaxies derived from numerical $\Lambda \mathrm{CDM}$ simulations is universal and scale invariant. Rotation curves are an important tool to put these predictions to the test. In order to obtain an extended rotation curve of the spiral galaxy NGC 755 we applied a new method to fit tilted rings to a data cube acquired with the VLA. Our findings were combined with existing results from optical spectroscopy to provide the highest possible spatial resolution towards the center. We present the resulting rotation curve and its implications for the density distribution of this galaxy's dark matter halo, indicating a core-dominated halo model.
\end{abstract}

Baryons in Dark Matter Halos

5-9 October 2004

Novigrad, Croatia

${ }^{*}$ Speaker. 


\section{Introduction}

According to today's standard cosmological view, the universe is dominated by dark energy $\left(\Omega_{\Lambda} \approx 0.7\right)$ and dark matter $\left(\Omega_{D M} \approx 0.3\right)$, and the structures in the universe arose from hierarchical clustering. Computer simulations of this cosmic evolution [9] allow to predict the dark matter density distribution in spiral galaxies, but the resulting cusp-dominated halos seem to disagree with core-dominated halo models which fit most observations best [2].

NGC 755 is a field galaxy of type SBb. Its distance to our solar system is $(21.3 \pm 1.6) \mathrm{Mpc}$, determined using the Tully-Fisher-relation [10]. 8 hours of HI observations were carried out with the VLA (C-configuration) in 2002. Our results were combined with existing results from optical spectroscopy to provide the highest possible spatial resolution towards the center. The resulting rotation curve - obtained using a new method to fit tilted rings to a data cube - allows us to analyze the density distribution of this galaxy's dark matter halo.

\section{HI data reduction}

The visibility dataset was flagged interactively and then processed by a newly developed data reduction pipeline based on the MIRIAD software package. This pipeline carries out (self-)calibration, continuum subtraction, inversion and iterative CLEANing [3] in a completely automated way and delivers data cubes of highest quality.

In order to improve the iterative CLEAN process, we included an own program in the MIRIAD environment. It provides the opportunity to push the clip level for the clean mask to very low values by erasing mask fields caused by noise peaks. Therefore the pipeline is especially sensitive to faint extended emission.

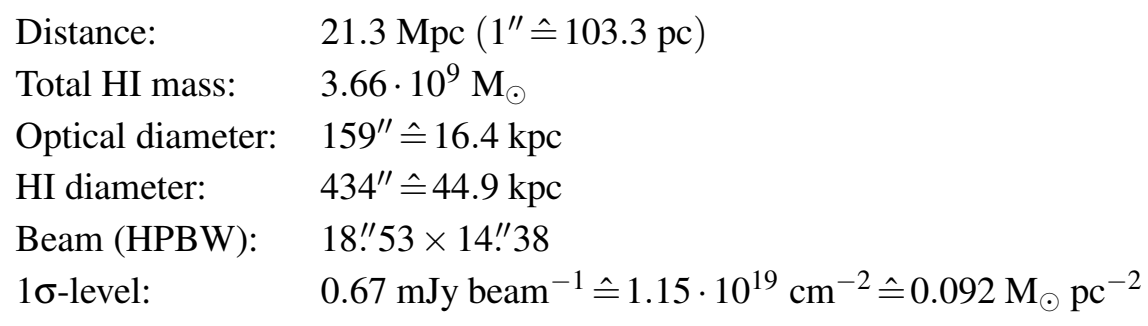

Table 1: Basic properties of NGC 755 and the obtained data

\section{Tilted-ring modelling}

Tilted-ring-models are used to parametrize warped disk galaxies. Each ring is characterized by inclination, position angle, rotation velocity and HI density. We obtained a first guess for the model parameters through a fit to an optimized velocity field. Then, we applied an iterative fitting method employing the GIPSY software package with its powerful GALMOD task. This method varies the parameters and compares the model datacube with the original one via a $\chi^{2}$-test until reaching the best-fit model. The best-fit model for NGC 755 is shown in Figure 1. 


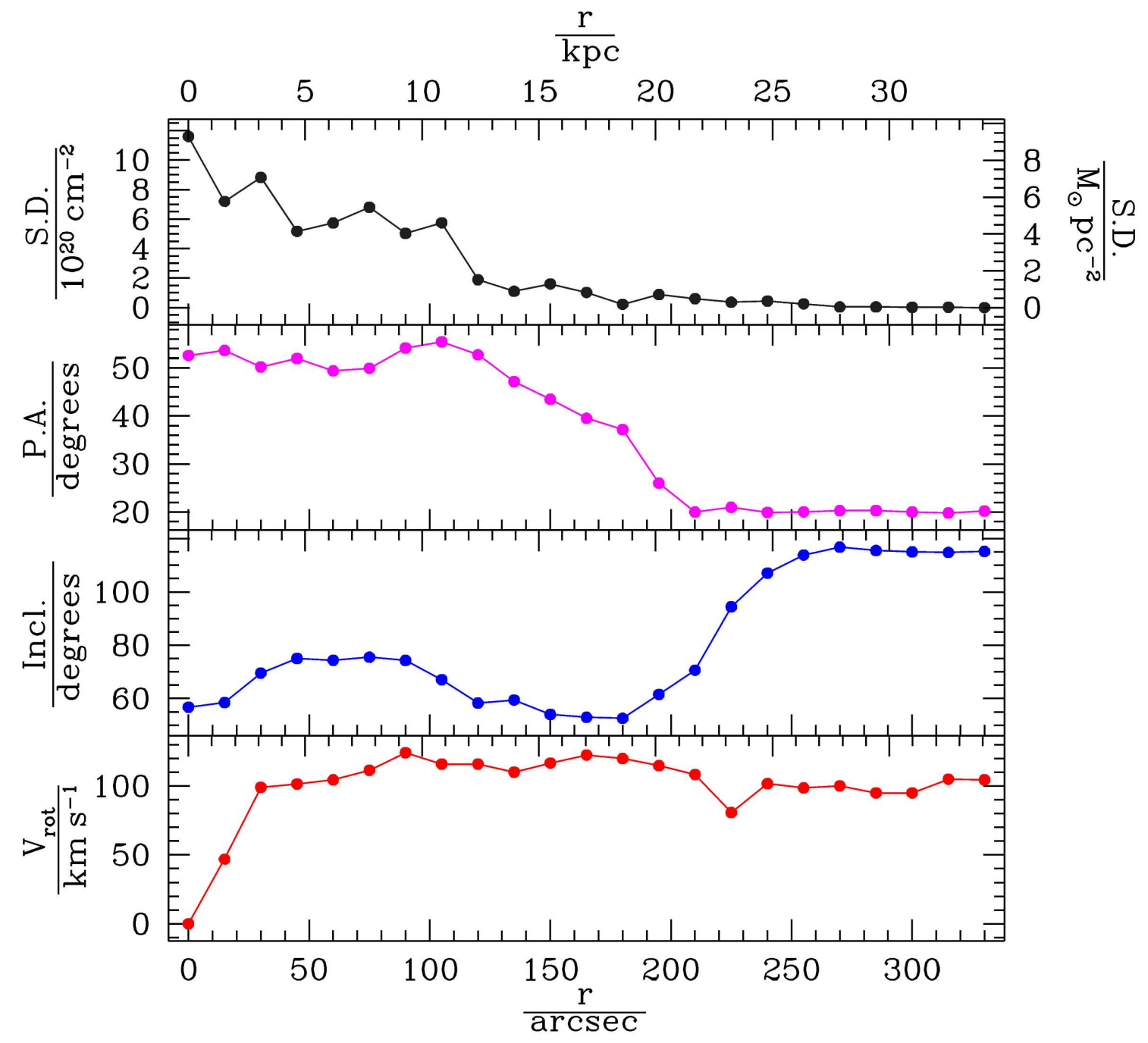

Figure 1: The derived parametrization of NGC 755: HI surface density (S.D.), position angle (P.A.), inclination (Incl.), and rotation velocity $\left(\mathrm{v}_{\mathrm{rot}}\right)$

\begin{tabular}{llcc} 
Fitted profile & Density function & Stellar M/L-ratio & Reduced $\chi^{2}$ \\
\hline Pseudo-isothermal halo & $\rho(r)=\frac{\rho_{0}}{1+\frac{r^{2}}{r_{0}^{2}}}$ & 0.26 & 5.88 \\
Burkert halo & $\rho(r)=\frac{\rho_{0} r^{3}}{\left(r-r_{0}\right)\left(r^{2}-r_{0}^{2}\right)}$ & 0.20 & 3.20 \\
NFW halo & $\rho(r)=\frac{\rho_{s}}{\frac{r}{r_{s}}\left(1+\frac{r}{r_{s}}\right)^{2}}$ & 0.21 & 8.89 \\
HI scaling & $\rho(r)=s \cdot \rho_{H I}(r)$ & 0.92 & 9.31 \\
\hline MOND & $g(r)=\frac{\sqrt{G \cdot M(r) \cdot a_{0}}}{r}$ & 0.69 & 8.87
\end{tabular}

Table 2: Best-fit parameters for different halo profiles and the corresponding reduced $\chi^{2}$ (Pseudo-isothermal and Burkert halo are core-dominated, NFW halo is cusp-dominated) 


\section{Mass decomposition}

The mass decomposition was performed using the GIPSY task ROTMAS. For the innermost part of the rotation curve we used more exact results [1] based on optical data [7]. The gaseous constituent of the mass-model was derived from the modelled HI surface densities assuming a flat HI disk, while the stellar component was assumed to be an exponential disk with a constant M/Lratio, which was left as a free parameter. Finally, the dark matter contribution was fitted with an analytic parametrized density profile (cusp- and core-dominated halo profiles as well as HI scaling). We also performed a mass decomposition without dark matter using MOND [8]. After that a $\chi^{2}$ test determined the best fitting theoretical prediction. As one can see in Table 2, the Burkert halo fits best for NGC 755 .

\section{Results and future work}

We developed an automated reduction pipeline to obtain high-quality data cubes, which is a powerful tool for fast but reliable data reduction.

We applied a new method to obtain reliable tilted-ring-models of HI galaxy disks by performing a $\chi^{2}$-fitting directly to the datacube. This method will be upgraded to allow for additional degrees of freedom (e.g. shifting of the kinematic center with radius).

Our mass decompositions of NGC 755 show that a core-dominated halo fits the rotation curve better than a cuspy halo does, well in agreement with previous studies (e.g. [5]).

\section{References}

[1] Borriello A. \& Salucci P., 2001, MNRAS, 323, 285

[2] Burkert A., 1995, ApJ, 447, 25

[3] Clark B.G., 1980, A\&A, 89, 377

[4] García Ruiz I., 2001, PhD thesis, Groningen

[5] Gentile G. et al., 2004, MNRAS, 351, 903

[6] Józsa G., 2002, Diploma thesis, Bonn

[7] Matthewson, Ford \& Buchhorn, 1992, ApJS, 81, 413

[8] Milgrom M., 1983, ApJ, 270, 365

[9] Navarro J., Frenk C. \& White S., 1996, ApJ, 462, 593

[10] Sakai S. et al., 2000, ApJ, 529, 698 